\title{
Refractory Celiac Disease New Diagnostic Approaches and Current Treatment
}

\author{
Michael Schumann*, Christian Bojarski, Verena Moos, Severin Daum \\ Medizinische Klinik für Gastroenterologie, Infektiologie und Rheumatologie, Charité, Campus Benjamin Franklin, Berlin, Germany \\ *Corresponding author: michael.schumann@charitte.de
}

Received March 21, 2014; Revised March 23, 2014; Accepted March 24, 2014

\begin{abstract}
Since refractory celiac disease (RCD) may cause severe complications and is associated with a poor prognosis in a portion of patients, early diagnosis and treatment is of importance. Current diagnostics include complex PCR-based molecular pathology and FACS techniques in order to differentiate type I and type II RCD, the latter being associated with the development of an enteropathy-associated $\mathrm{T}$ cell lymphoma (EATL). Recently established treatment options also include high-dose chemotherapeutic regimens for RCD type II which have significantly improved survival. However, treatment strategies are highly individual targeting also complications of malabsorption and should be started in the RCD phase of the disease. Therefore, adequate diagnostics are pivotal for patient outcome.
\end{abstract}

Cite This Article: Michael Schumann, Christian Bojarski, Verena Moos, and Severin Daum, "Refractory Celiac Disease New Diagnostic Approaches and Current Treatment.” International Journal of Celiac Disease, vol. 2, no. 1 (2014): 33-37. doi: 10.12691/ijcd-2-1-10.

\section{Introduction}

Refractory celiac disease (RCD) develops in0.7-1.5\% of patients with celiac disease (West et al., Roshan et al.).It is clinically defined by amalabsorptive state secondary to a celiac enteropathy (Marsh IIIA or more) and arises although the patient is on a strict gluten-free diet (GFD) forat least one year (Cellier et al., 2000, Nijeboer et al., 2013, Rubio-Tapia et al., 2010). Comprehensive clinical diagnosis of RCD requires (i) review of the patient's diet (best done by a trained dietician) and (ii) the exclusion of differential diagnoses of malassimilation including inflammatory bowel disease, microscopic colitis, autoimmune enteropathy, tropical sprue, lambliasis, HIV enteropathy, Whipple's disease, common variable immunodeficiency, bacterial overgrowth, exocrine pancreatic failure, hypergastrinemia/ZollingerEllison syndrome and irritable bowel syndrome. Of note, a high percentage of patients that were initially suspected to have fallen ill of RCD turn out to be exposed to small amounts of gluten secondary to low adherence to the GFD(Abdulkarim et al., 2002). However, once a diagnosis of RCD is firmly established, further diagnostic procedures are needed to decipher the RCD subtype and to define the extent of disease.

\section{Pathophysiology of RCD and natural Course of Disease}

Based on current knowledge the entity of RCD is subclassified in two subtypes, type I and type II. While RCD type II is associated with a clonal proliferation of aberrant intraepithelial lymphocytes (IELs) and a high risk of the disease progressing to an enteropathy-associated $\mathrm{T}$ cell lymphoma (EATL), RCD type I corresponds to villous atrophy in the absence of clonal IEL proliferation and is in most cases not associated with lymphoma development (Cellieret al., 2000\&Daum et al., 2001). Although the immunopathology of the two subtypes appears to be unrelated, similar mucosal lesions and barrier defects might develop (Schumann et al., 2012).

\subsection{RCD Type I}

Apart from rare cases where patients initially diagnosed as RCD type I later developed RCD type II and EATL the risk of developing a $\mathrm{T}$ cell lymphoma is comparatively low. Thus the 5-year survival rate of RCD type I is fairly high (90\%), however patients often suffer from thromboembolic and infectious complications (Daum et al., 2009).

Mechanisms that induce a diet-refractory state in RCD type I remain mostly elusive. One association is found with collageneous sprue, where a thickened subepithelial collagen layer might cause a persistent mucosal lesion even after gluten as the initial trigger was disbanded from the daily diet (Daum et al., 2006 \&Vakiani et al., 2010). Moreover, Caruso et al. revealed expression of TNF- $\alpha$ and interleukin-6 (IL-6) in duodenal mucosa of RCD type I, data being further substantiated by the successful treatment of an RCD type I patient with the TNF- $\alpha$ antibody infliximab (Caruso et al., 2014).However, it is believed that RCD type I might rather be a heterogeneous group of underlying pathophysiologies that cannot be explained with a single mechanistic scenario.

\subsection{RCD Type II}


Secondary to the pronounced malabsorption syndrome and the high risk of developing an EATL in the course of the disease, the prognosis of RCD type II is significantly worse than that of RCD type I with a 5-year survival rate of 53\% (Daum et al., 2009). Deduced from epidemiologic studies examining the rate of lymphoma development and from the notion that RCD type II is most often found in individuals being 50 years of age or older (Malamut et al.) it was suspected that a long-standing mucosal lesion especially in cases not adherent to the GFD - might be an independent risk factor for the development of RCD type II (Holmes et al.).

The cell type in the center of immunopathology in RCD type II is a population of aberrant intraepithelial lymphocytes (IEL). Comprehensive research by a number of groups has assembled a pathogenetic model, whereby interleukin-15 (IL-15), presumably produced by enterocytes, acts as a major survival factor for IELs in general, but specifically for aberrant IELs in RCD type II, an effect that is secondary to a considerable downregulation of IEL apoptosis by IL-15, which can be blocked by application of an IL-15 antibody (Mention et al., 2003 \&Malamut et al., 2010). IL-15 also drives IL-21 production in lamina propria lymphocytes in celiac small intestinal mucosa (Sarra etal.), which had previously been shown to synergize on T cells and on natural killer(NK-) cells. In this context the association of the IL2/IL21 locus with celiac disease by genome-wide association studies is worth mentioning(van Heel et al., 2007). Moreover, IL-15 drives NKG2D-mediated cytotoxicity of IELs against enterocytes in RCD presumably by transcriptional regulation of the NKG2D ligand MIC-A in duodenal epithelial cells (Hue et al.). Interestingly, a recent study designed to identify the presumed precursor cell came up with a novel lymphocytic cell type (lineage $e^{-} \mathrm{CD}^{+}$, CD127- CD34- and IL15 receptor- $\beta^{+}$), that was not only

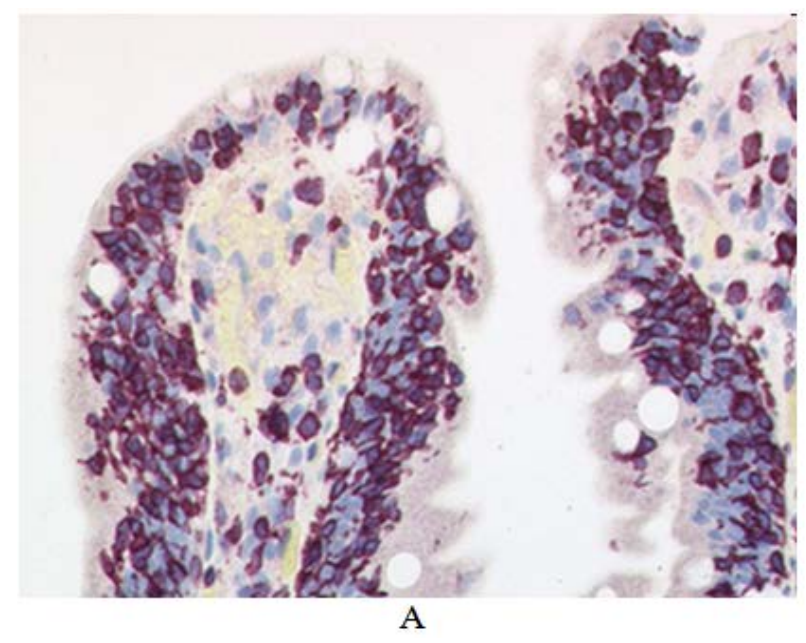

extracted from RCD type II duodenal mucosa but was also found insmall intestinal mucosa of healthy individuals (Schmitz et al.). This study is part of a continuing discussion on the origin of RCD type II IELs, that show T cell similarities (clonal TCR rearrangement, intracellular $\mathrm{CD} 3 \varepsilon$ ) on the one hand, but NK cell properties (NK-like cytotoxicity) on the other hand (Malamut et al., 2012).Another interesting piece of recent research mechanistically clarified the intracellular retention of CD3eas well as $\mathrm{T}$ cell receptor chains, a phenomenon that clinicians made use of by introducing FACS techniques to identify aberrant IELs by their intracellular CD3 (Verbeek et al.). Tjon et al. uncovered an intracellular assembly defect of the CD3-TCR complex in aberrant IELs, that impedes the physiological shuttling of this complex to the cell membrane of $\mathrm{T}$ cells (Tjon et al.).

\section{Diagnostics in RCD}

\subsection{Conventional Immunohistochemical and Molecular Pathology to Decipher the RCD Subtype}

Histology of the small intestine (most often duodenum, after enteroscopy also jejunum orileum) reveals villous atrophy and crypt hyperplasia, as well as an intraepithelial lymphocytosis. An important next diagnostic step is to do immunohistochemistry with staining of CD3, CD4 and CD8, which in the case of RCD type II reveals loss of the CD8 antigen in $>50 \%$ of CD3-positive, CD4-negative $\mathrm{T}$ cells (Figure 1). Unspecific CD8 antigen loss might be found in uncomplicated celiac disease in mucosae with a high proportion of $\gamma \delta \mathrm{T}$ cells, since these $\mathrm{T}$ cells physiologically lack CD8 (Daum et al., 2001 \& Liu et al., 2010 \&Verkarre et al., 2003).

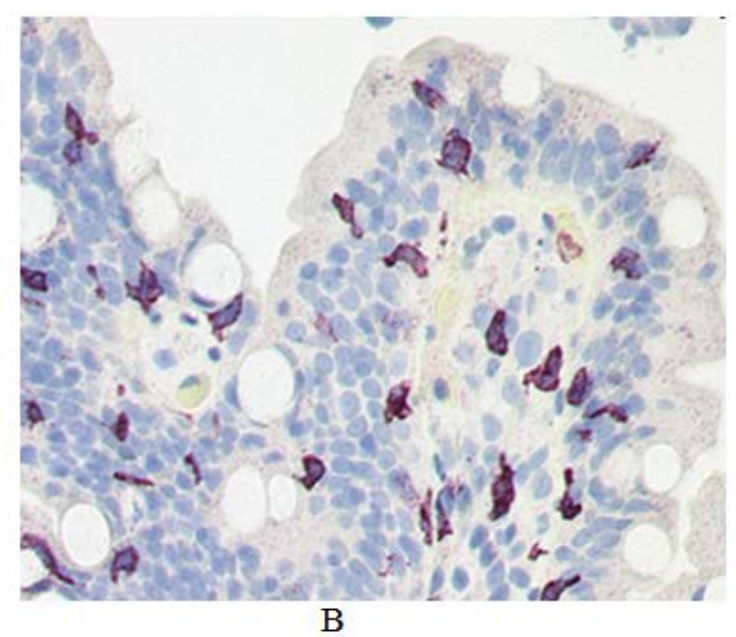

Figure 1. Immunohistochemistry as found in refractory celiac disease type II. A: CD3 staining. B: CD8 staining

The immunohistochemistry is complemented by molecular pathology analysisto identify clonal Tcell population of IELs. This is achieved by multiplex PCR employing primers for all known TCR- $\gamma \mathrm{V}$ - and Jsegments. Due to the diversity of the individual and highly variable CDR3 regions of the $\mathrm{T}$ cellreceptor (TCR)- $\gamma$ chain, complex mixture of PCR products is generated which can be analysed by high resolution GeneScan technology. This enables the detection of even small populations of clonal IELs in the small intestinal mucosa (Figure 2). In a healthy individual this analysis yields a polyclonal array of PCR products with a Gaussian-like distribution of their length. However, in RCD type II, a monoclonal pattern is found in many cases with a dominant peak representing a single TCR- $\gamma$ rearrangement of clonally proliferating $\mathrm{T}$ cells. This rearrangement is reproducible, i.e. when small intestinal mucosa of the same patient is examined at a later point in time the 
identical TCR rearrangement will re-appear. In other cases, oligoclonal rearrangement patterns (i.e. several dominant
PCR products) are present, which might in some cases impede the diagnostic decision process(Daum et al., 2001).
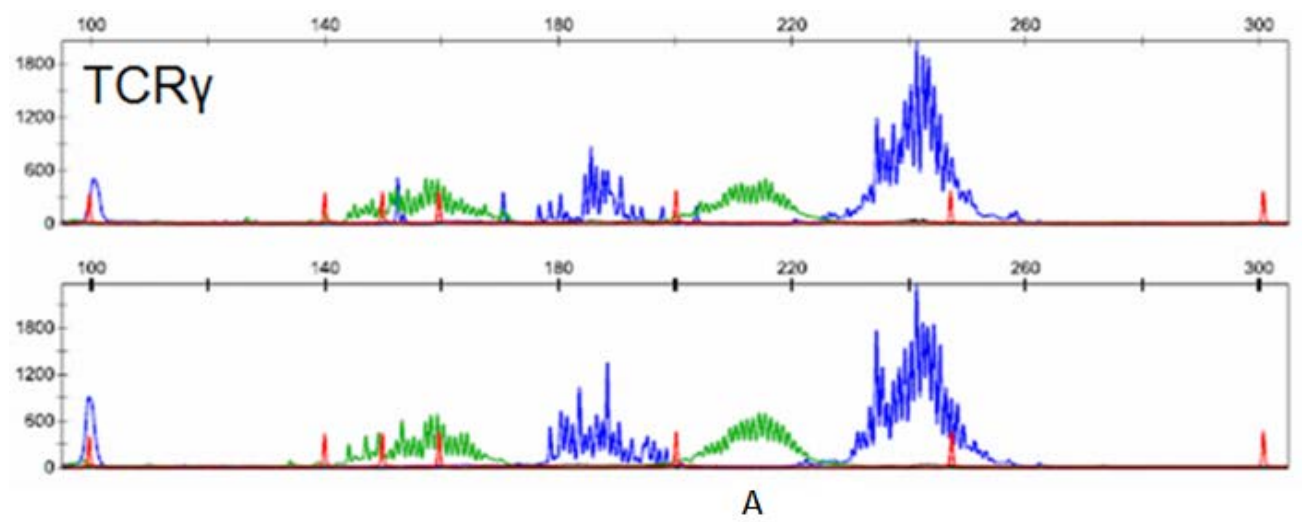

\section{polyclonal}

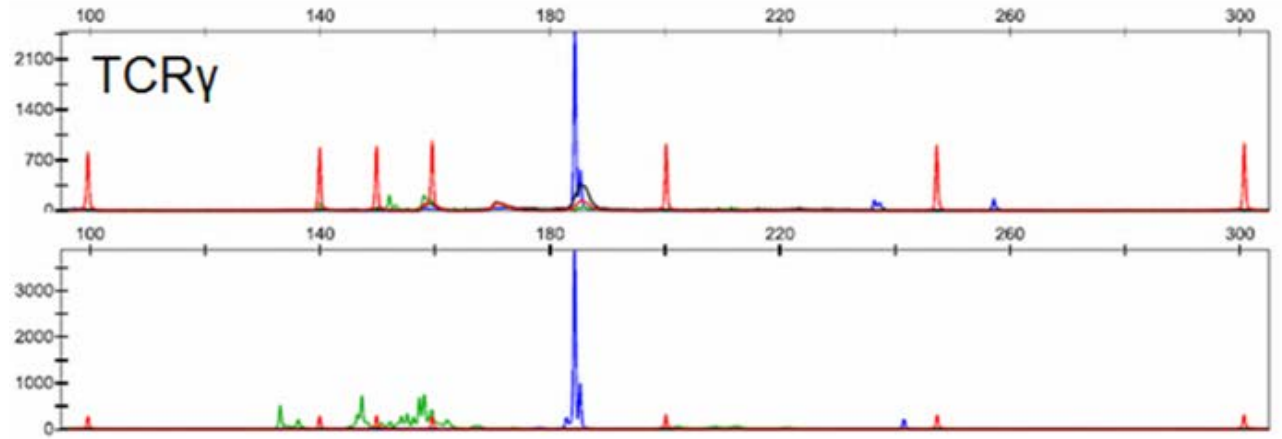

monoclonal

B

Figure 2. GeneScan analysis of T cell receptor (TCR)-gamma gene rearrangements. PCR was done using two sets of primers (set A and set B) covering all possible TCR-gamma rearrangements. Due to a different fluorescence labeling of the J-gamma gene segment families, the type of J-segment involved in the rearrangement can be identified (blue and green). Size markersare highlighted in red. A: polyclonal TCR-gamma gene rearrangement. B: Monoclonal TCR-gamma gene rearrangement(Courtesy of Professor M. Hummel, Molecular Pathology Unit, Charité)

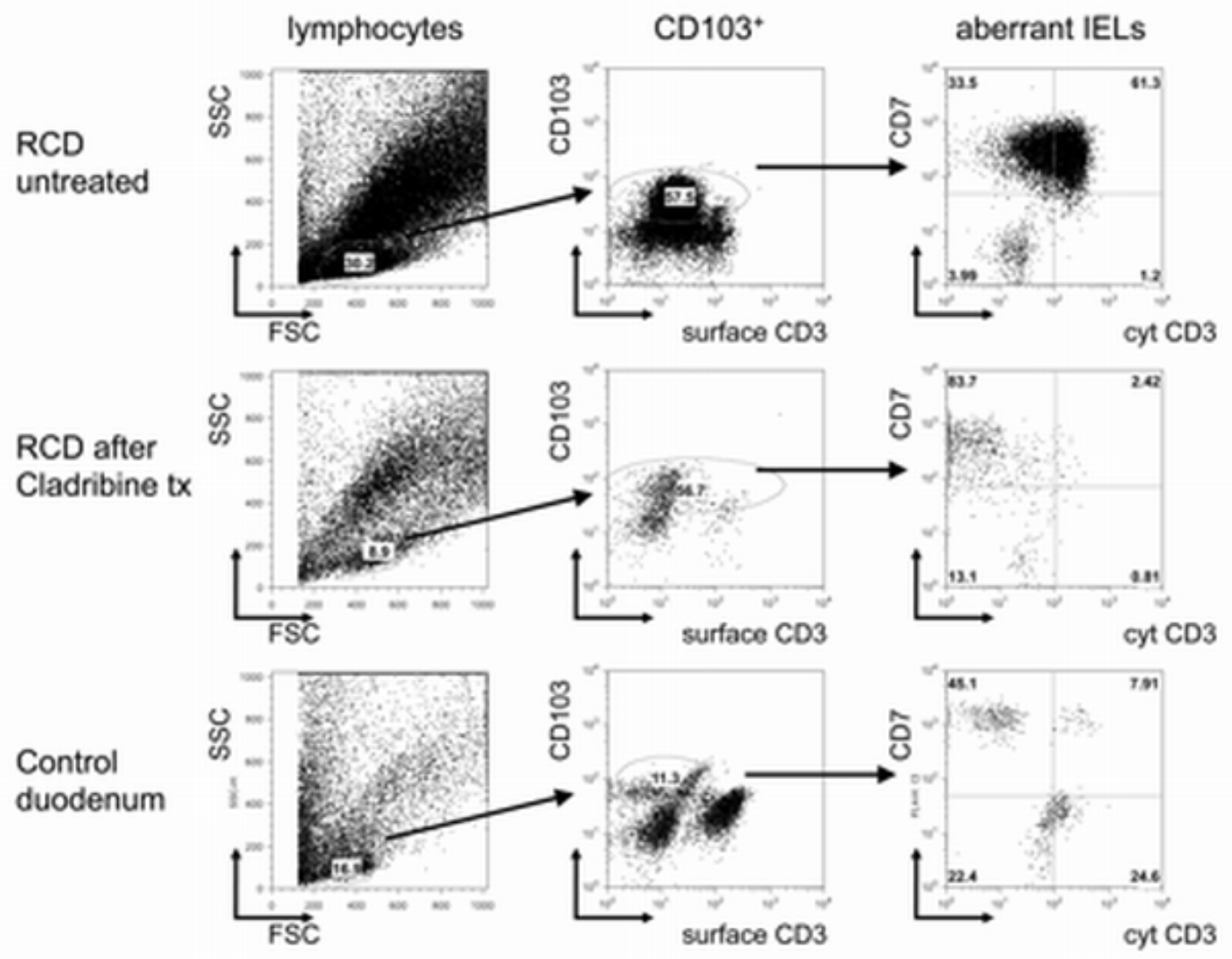

Figure 3. FACS analysis of isolated IELs from duodenal mucosabiopsies. Forward andsidewardscatter (FSC, SSC) wereusedtogateforlymphocytes. $\mathrm{CD}_{103}{ }^{+}$, surCD3 3 cellsweregatedandanalyzedfor CD7 and cytoplasmic-CD3 expression. Upperpanel: duodenal mucosaof a patientwith RCD type II (61\% aberrant IELs). Middlepanel: Same patient after treatmentwirthcladribine (3\% aberrant IELs). Lowerpanel: Duodenal mucosaof a healthy individual (8\% aberrant IELs) 


\subsection{Alternative Diagnostic Procedures to Subtype RCD}

Various research groups have introduced FACS analysis of isolated IELs as a more accurate technique to classify RCD as either type I or type II (Verbeek et al., 2008\&Cellier et al., 1998 \&Cellier et al., 2000). This technique involves isolation of IELs from endoscopic small intestinal biopsy specimen in a Calcium ionchelating buffer (containing EDTA or EGTA)followed by fixation of IELs. If staining of intracellular proteins is intended, cells are additionally permeabilized. After immunostaining of cells with fluorescently labeled antibodies flow cytometric analysis is done using a Fluorescence Activated Cell Scanner (FACS) (Figure 3). Using this approach RCD type II was associated with a specific (so-called 'aberrant') IEL phenotype, exhibiting cytoplasmic $\mathrm{CD}^{+}, \mathrm{CD}^{+}$, surface $\mathrm{CD}^{-}$,surface $\mathrm{TCR}^{-} \mathrm{T}$ cells. Verbeek et al. evaluated $>20 \%$ of aberrant IELs to be diagnostic for RCD type II.

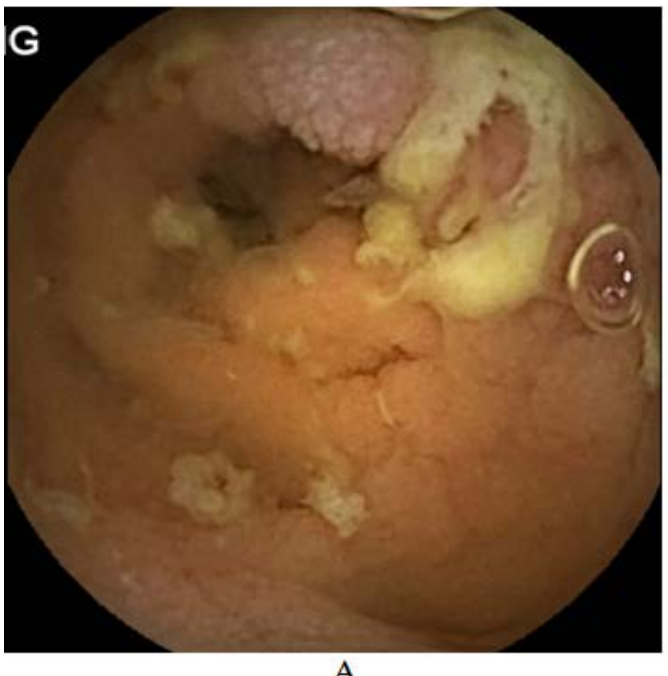

\subsection{Diagnostic Procedures to Define the Extent of Disease}

Since aberrant IELs can disseminate to the peripheral blood, to other parts of the GI tract (stomach, colon) and other epithelial organs (e.g. skin) during disease progression from RCD to overt EATL, further diagnostic procedures need to consider these locations. Therefore a work-upincludes a thorough inspection of the patient, a peripheral blood smear, gastroscopy and a colonoscopy. Histologic examination of the patient's bone marrow is done to exclude bone marrow infiltration. Further steps in our department includean MRI scan focusing on the small intestine and abdominal lymph nodes followed by video capsule endoscopy. If these techniques yield results suspicious for small intestinal lymphoma, an enteroscopy (single or double balloon) is performed to collect biopsies for further (immuno-)histologic characterization or molecular pathology analysis (Figure 4).

Figure 4. Enteroscopy in celiac disease. A: Video capsule endoscopy showing ulcerative jejunitis in a patient with RCD type II. B: Double balloon enteroscopy ascertained diagnosis of EATL in a patient previously being diagnosed as RCD type II

The literature reveals alternative imaging strategies: Stijn et al. have established an MRI enteroclysis protocol that appears to specifically recognize alterations associated with RCD type II and includes scarcity of jejunal folds, mesenteric fat infiltration, and bowel wall thickening (Stijn et al., 2010). However, video capsule endocopywasalso successfully performed by a number of clinical research groups and appears to be optimal to reveal small ulcerative mucosal lesions as found in ulcerative jejunitis (Daum et al., 2007\&Collin et al., 2012 \&Barret et al., 2012). Double balloon enteroscopy was also studied and proved to be suitable especially in establishing lymphoma diagnosis on the basis of jejunal or ileal histology (Hadithi et al., 2007). Those studies that included patients with RCD type I could not find abnormalities in the small intestine in these patients.

\subsection{Use of Serology in Refractory Celiac Disease}

Transglutaminase serology (Tg-IgA or -IgG) is usually required to be negative. Positive Tg-serology should prompt the clinician torule out lack of adherence to the

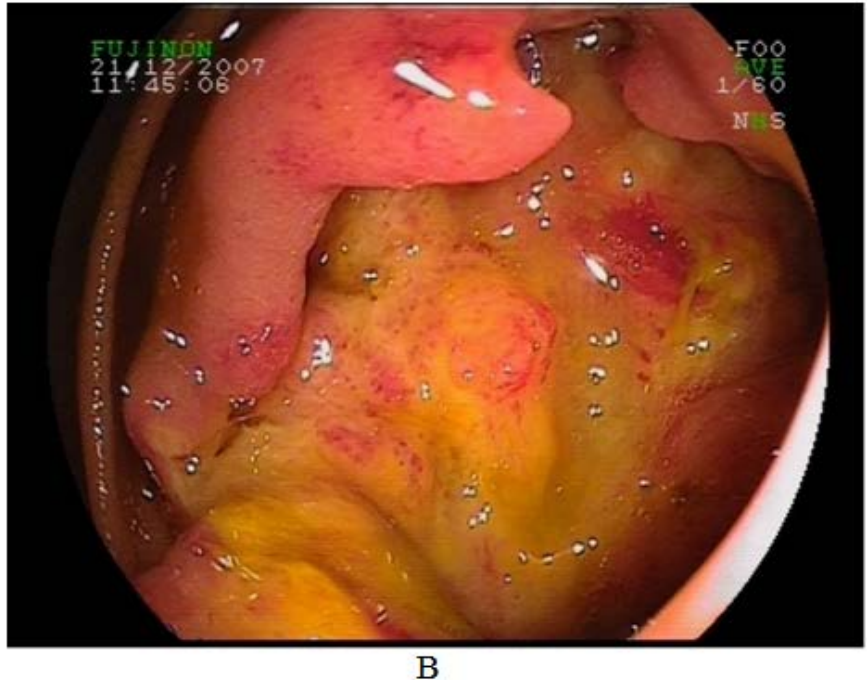

GFD, since antibody production is usually triggered by exposure to antigenic gliadin (Rubio-Tapia et al., 2010). However, a few cases (13-19\% are reported) with credible adherence to the GFD and elevated Tg-serology are found in most collectives of RCD patients (Malamut et al., 2009\& Rubio-Tapia et al., 2009). Thus, classical (i.e. native) gliadin antibodies might be of some use in RCD patients to confirm the lack of gluten contamination in the patient's diet, since these antibodies reflect gluten exposure in celiac patients. This is of special interest in those rare cases, where transglutaminase serology is still moderately elevated, which might not reflect a continuing exposure to gluten but rather a non-specifically elevated serology.

\section{Treatment Options}

For a more elaborate presentation of current treatment modalities we refer to excellent recent reviews on this topic (e.g. Nijeboer et al., 2013).Continuing the GFD is pivotal to the patients further prognosis even if the capability of the diet to positively influence disease course 
was not clearly visible to patient and doctor. In both entities a malabsorption syndrome often is the dominating clinical problem. Thus, nutritional support is one of the first measures that need to be considered. Treatment strategies beyond nutritional help differ substantially in the two RCD subtypes due to the different immunopathology and associated prognosis of disease.

\subsection{RCD Type I}

In RCD type I the first line of immunosuppressive treatment is the oral glucocorticoid budesonide given as a formulation that releases the drug along the small intestine (Daum et al., 2006).Once an effect is achieved tapering down the budesonide dose to see if effects on villous atrophy-associated malabsorption continue even in the absence of further immunosuppression is one option. Should symptoms reappear, reinduction with budesonide can be done followed by a parallel introduction of azathioprine to achieve a sustained treatment effect. It is reported that in some patients (while azathioprine is continued) corticoids later on can be tapered (Goerres et al., 2003). Another treatment option is small intestinal release mesalamine, which seems to be active in RCD type I given either as a stand-alone medication or parallel to oral budesonide treatment (Jamma et al., 2012).Recently, tioguanine was studied in 12 RCD type I patients resulting in a clinical and also histological response in $80 \%$ of the patients. However, one patient died secondary to disease progression four months after treatment was started (Tack et al., 2012). Single reports about successful immunosuppressive treatment with the TNF- $\alpha$ antibody infliximab but also tacrolimusexist (e.g. Caruso et al.).

\subsection{RCD Type II}

In a first phase of RCD type II, budesonide helps stabilizing the malabsorptive syndrome and frequently leads to a significant reduction in stool frequency. Parallel monitoring of duodenal mucosa reveals that most often Marsh stage is unchanged although a clinical benefit clearly was noted (Daum et al., 2006). Once disease progresses further treatment is required, which cannot be immunosuppressive, since this was shown to be ineffective (Goerres et al., 2003). At this stage, treatment strategy switches to a chemotherapeutic therapy regimen with the purine analogue cladribine being the first line of treatment as it combines effective treatment on lymphocytes with only modest adverse effects and is therefore specifically useful with RCD type II as this disease is typically found in an older patient population. Another treatment option is autologous stem cell therapy (auto-SCT) preceded by conditioning with fludarabine and melphalan. This regimen was shown in 13 patients to induce remission in all patients with a relapse in one patient and a fatal complication of auto-SCT in another patient (Tack et al., 2011). Future treatment options that need to be studied include IL-15 antibody treatment which might be effective secondary to induction of apoptosis in aberrant IELs.

\section{References}

[1] West J et al., Gastroenterology 2009; 136(1): 32-34.

[2] Roshan B et al., Am J Gastroenterol2011; 106(5): 923-928.

[3] CellierC et al., Lancet. 2000; 356(9225): 203-208.

[4] Nijeboer P et al., Gastroenterol Res Pract. 2013; 518483.

[5] Rubio-Tapia et al., Gut. 2010; 59(4): 547-557.

[6] Abdulkarim et al., Am J Gastroenterol.2002; 97(8): 2016-2021.

[7] Daum S et al., Clin Gastroenterol Hepatol 2006; 4(10): 1232-1236.

[8] Vakiani E et al., Mod Pathol. 2010 Jan; 23(1):12-26.

[9] Caruso R et al., Clin Sci (Lond). 2014; 126(6): 451-458.

[10] Sarra et al., Mucosal Immunol. 2013; 6(2): 244-255.

[11] Holmes GK et al., Gut. 1989; 30(3): 333-8.

[12] Schumann M et al., Ann N Y AcadSci. 2012 Jul;1258:43-51

[13] Daum S et al., Eur J Gastroenterol Hepatol. 2009; 21(1): 66-70.

[14] Mention JJ et al., Gastroenterology. 2003; 125(3): 730-745.

[15] Malamut G et al., Gastroenterology. 2009;136(1):81-90.

[16] Malamut G et al., J Clin. Invest. 2010; 120(6): 2131.

[17] Van Heel et al., Nat Genet. 2007;39(7): 827-829.

[18] HueS et al., Immunity 2004; 21: 367-377.

[19] Schmitz et al., Gut 2013; 62: 509-519.

[20] Malamut et al., SeminImmunopathol 2012; 34: 601-613.

[21] Verbeek et al., Clinical Immunology 2008; 126: 48-56.

[22] Tjon et al., Blood 2012; 112(13): 5103-5110.

[23] Daum S et al., Gut 2001; 49(6): 804-812.

[24] Liu H et al., Gut 2010; 59: 452-460.

[25] Verkarre V et al., Gut 2003; 52(2): 205-211.

[26] Verbeek WH et al., Clinical Immunology 2008; 126, 48-56.

[27] Cellier C et al., Gastroenterology 1998; 114(3): 471-481.

[28] Stijn et al., Radiology2011; 259(1): 151-161.

[29] Daum S et al., Endoscopy 2007; 39(5): 455-458.

[30] Collin P et al., J Dig Dis. 2012; 13(2): 94-99.

[31] Barret et al., Am J Gastroenterol. 2012; 107(10): 1546-1553.

[32] Hadithi et al., Am J Gastroenterol. 2007; 102(5): 987-996.

[33] Malamut G et al., Gastroenterology 2009; 136: 81-90.

[34] Rubio-Tapia A et al., Gastroenterology 2009; 136(1): 99-107.

[35] Daum S et al., Digestion 2006; 73(1): 60-68.

[36] Goerres MS et al., Aliment Pharmacol Ther. 2003; 18(5): 487-494.

[37] JammaS et al., J Clin Gastroenterol 2010; 45(1): 30-33.

[38] Tack GJ et al., Aliment Pharmacol Ther. 2012; 36(3): 274-281.

[39] Tack GJ et al., Bone Marrow Transplant 2011; 46(6): 840-846. 